PROCEEDINGS OF THE

AMERICAN MATHEMATICAL SOCIETY

Volume 134, Number 3, Pages 859-869

S 0002-9939(05)08118-9

Article electronically published on July 18, 2005

\title{
ON MULTIVARIATE SUBDIVISION SCHEMES WITH NONNEGATIVE FINITE MASKS
}

\author{
XINLONG ZHOU
}

(Communicated by David R. Larson)

\begin{abstract}
We study the convergence of multivariate subdivision schemes with nonnegative finite masks. Consequently, the convergence problem for the multivariate subdivision schemes with nonnegative finite masks supported on centered zonotopes is solved. Roughly speaking, the subdivision schemes defined by these masks are always convergent, which gives an answer to a question raised by Cavaretta, Dahmen and Micchelli in 1991.
\end{abstract}

\section{INTRODUCTION}

Subdivision schemes provide important techniques for fast generation of curve and surfaces. A recursive refinement of a given control points will lead in the limit to a desired visually smooth object. This method also plays an important role in wavelet analysis.

Denote $\mathbb{Z}^{s}$ the integer lattice. A subdivision scheme is defined by a fixed finitely supported real sequence (mask) $a=\left\{a_{\alpha}: \alpha \in \mathbb{Z}^{s}\right\}$. Associated with this mask is the Laurent polynomial

$$
a(z)=\sum_{\alpha} a_{\alpha} z^{\alpha}
$$

where $z=\left(z_{1}, \ldots, z_{s}\right)^{T} \in \mathbb{R}^{s}$ and $z^{\alpha}=z_{1}^{\alpha_{1}} \ldots z_{s}^{\alpha_{s}}$ for $\alpha=\left(\alpha_{1}, \ldots, \alpha_{s}\right)^{T}$. Given an initial finite sequence of data values, $v^{0}=\left\{v_{\alpha}^{0}\right\}$, a subdivision scheme with mask $a$ defines recursively new sequence of value $v^{k}$ by applying the rule

$$
v_{\alpha}^{k}=\sum_{\beta} v_{\beta}^{k-1} a_{\alpha-2 \beta} .
$$

This scheme is said to be convergent if for each $v^{0}$ there exists a continuous function $f_{v}$ such that

$$
\lim _{k \rightarrow \infty} \sup _{\alpha}\left|f_{v}\left(\frac{\alpha}{2^{k}}\right)-v_{\alpha}^{k}\right|=0
$$

and $f_{v} \not \equiv 0$ for at least one $v$. Moreover the limit function $f_{v}$ has the representation

$$
f_{v}(x)=\sum_{\alpha} v_{\alpha}^{0} \varphi(x-\alpha)
$$

Received by the editors March 22, 2004 and, in revised form, October 8, 2004.

2000 Mathematics Subject Classification. Primary 65D17, 26A15, 26A18.

Key words and phrases. Nonnegative mask, subdivision scheme, zonotope.

(C)2005 American Mathematical Society Reverts to public domain 28 years from publication 
where $\varphi$ is the refinable function which is the unique compactly supported solution of the functional equation

$$
\varphi(x)=\sum_{\alpha} a_{\alpha} \varphi(2 x-\alpha)
$$

and the support is the convex hull of $\left\{\alpha: a_{\alpha} \neq 0\right\}$ (see [2]). In fact, $\varphi$ is the function obtained by subdivision from the initial data $v_{\alpha}^{0}=\delta_{0, \alpha}$. On the other hand, $\varphi$ can also be obtained by the so-called cascade algorithms. Thus, beginning with $\varphi_{0}\left(x_{1}, \ldots, x_{s}\right)=h\left(x_{1}\right) \cdots h\left(x_{s}\right)$, where $h(y)=1-|y|$ if $|y| \leq 1$ and zero otherwise, one recursively defines

$$
\varphi_{k}(x)=\sum_{\alpha} a_{\alpha} \varphi_{k-1}(2 x-\alpha) .
$$

It is known that the uniform convergence of $\varphi_{k}$ is equivalent to the convergence of the corresponding subdivision scheme. A comprehensive discussion of this subject can be found in [2].

To describe the necessary and sufficient conditions of the convergence of the above present schemes we denote $a_{\alpha}^{k}=\sum_{\beta} a_{\beta}^{k-1} a_{\alpha-2 \beta}$ with the understanding $a_{\alpha}^{1}=a_{\alpha}$. It is easy to check that $a_{\alpha}^{k}$ are the coefficients of Laurent polynomial $\prod_{l=0}^{k-1} a\left(z^{2^{l}}\right)$, where, $z^{\mu}=z_{1}^{\mu} \cdots z_{s}^{\mu}$ if $\mu \in \mathbb{R}$. Denote further $E_{s}=\left\{\left(\beta_{1}, \ldots, \beta_{s}\right)\right.$ : $\left.\beta_{i} \in\{0,1\}\right\}$ as the set of extreme points of $(0,1)^{s}$. It is known (see e.g. [4, 6, 12]) that the following holds.

Theorem 1.1. A subdivision scheme associated with a fixed finitely supported real sequence (mask) $a=\left\{a_{\alpha}: \alpha \in \mathbb{Z}^{s}\right\}$ converges if and only if

$$
\sum_{\beta} a_{\alpha+2 \beta}=1, \quad \forall \alpha \in \mathbb{Z}^{s},
$$

and

$$
\lim _{k \rightarrow \infty} \sup _{\alpha \in \mathbb{Z}^{s}, e \in E_{s}}\left|a_{\alpha}^{k}-a_{\alpha-e}^{k}\right|=0 .
$$

The first condition is clear and easy to check. However, the second one is rather difficult to verify. Denote

$$
\rho(\Delta a)=\lim _{k \rightarrow \infty} \sup _{\alpha \in \mathbb{Z}^{s}, e \in E_{s}}\left|a_{\alpha}^{k}-a_{\alpha-e}^{k}\right|^{\frac{1}{k}} .
$$

One can show that the condition (1.4) is equivalent to $\rho(\Delta a)<1$, while $\rho(\Delta a)$ equals to the so-called joint spectral radius of some square matrices (see [3, 2, 6]). Some partial results concerning the computation of $\rho(\Delta a)$ can be found in [1, 4] and the papers cited there. On the other hand, by a result in [10] the calculation of the joint spectral radius is in general NP-hard. Therefore, it is useful in practice to find some classes of masks, for that we can simply determine whether $\rho(\Delta a)<1$ for the given mask $a$. In this paper we focus on subdivision schemes associated with nonnegative finite masks, a class possessed by many applications in geometric modelings. A remarkable fact of this class is that the convergence does not rely on the actual values of the mask but rather on the support of the mask, i.e., $\left\{\alpha: a_{\alpha} \neq 0\right\}$ (see [9, 7]). Recently, the author shows in [13] that for the univariate case (i.e. $s=1)(1.4)$ can be replaced by some very simple conditions. Thus, the following conjecture raised in $[8]$ is confirmed. 
Theorem 1.2. The univariate subdivision scheme with the nonnegative mask $a=$ $\left\{a_{0}, \ldots, a_{N}\right\}$, which satisfies $a_{0}, a_{N} \neq 0$, converges if and only if

1) $\sum_{j} a_{2 j}=\sum_{j} a_{2 j+1}=1$ and $0<a_{0}, a_{N}<1$,

2) the greatest common divisor of $\left\{j: a_{j} \neq 0\right\}$ is 1 .

An immediate consequence is that for any nonnegative mask $\left\{a_{0}, \ldots, a_{N}\right\}$, which satisfies the sum rule $\sum_{j} a_{2 j}=\sum_{j} a_{2 j+1}=1$ and $0<a_{0}, a_{N}<1$, the functional equation (1.1) always has a continuous nontrivial solution. Indeed, let $d=$ $\operatorname{gcd}\left(i: a_{i} \neq 0\right)$. Then $a_{i} \neq 0$ if and only if $i=d j$ for some $j$. The mask $b_{j}=a_{d j}$ satisfies 1) and 2) of Theorem 1.2. We therefore have a continuous function $\varphi$ for (1.1) with the mask $\left\{b_{j}\right\}$. Set $g(x)=\varphi(x / d)$. We obtain

$$
g(x)=\sum_{j} a_{j} g(2 x-j) .
$$

The aim of this paper is to study the convergence of multivariate subdivision schemes with nonnegative masks, including the schemes whose supports are centered zonotopes introduced in [2. In this case we will prove that the sum rule (1.3) ensures the convergence (see Theorem 2.5), which gives an answer to a question raised by Cavaretta, Dahmen and Micchelli in 1991 (see p. 55 of [2]).

\section{MAIN RESULTS}

To present our main results we introduce some more notations. For $x \in \mathbb{Z}^{s}$ we should denote $(x)_{j}, j=1,2, \ldots, s$, to be the $j$-th coordinate of $x$. Let $\mathcal{K} \subset \mathbb{Z}^{s}$ be a finite set and let $(\mathcal{K})_{j}=\left\{(x)_{j}: x \in \mathcal{K}\right\}, j=1,2, \ldots, s$. Further, denote $\min x_{j}=\min _{x \in \mathcal{K}}(x)_{j}$ and $\max x_{j}=\max _{x \in \mathcal{K}}(x)_{j}$.

Definition 2.1. A finite set $\mathcal{K} \subset \mathbb{Z}^{s}$ has property $\mathrm{P}$ if for $j=1,2, \ldots, s$ either $\min x_{j}+\max x_{j}$ is even and there is an $x^{\prime} \in(\mathcal{K})_{j} \backslash\left\{\min x_{j}, \max x_{j}\right\}$ so that $x^{\prime}-$ $\min x_{j}$ is odd or else there are $x^{\prime}, y^{\prime} \in(\mathcal{K})_{j} \backslash\left\{\min x_{j}, \max x_{j}\right\}$ such that $x^{\prime}+y^{\prime}$ is odd.

For $s=1$ Theorem 1.2 implies that the support of any convergent subdivision scheme with a finite nonnegative mask has property P. Conversely, if a finite set $\mathcal{K}=\left\{p_{0}, \ldots, p_{k}\right\} \subset \mathbb{Z}$ has property $\mathrm{P}$, we can find a nonnegative mask $\left\{a_{j}\right\}$ whose support is $\mathcal{K}$. Moreover, $0<a_{p_{0}}, a_{p_{k}}<1$, and the sum rule is fulfilled, i.e. $\sum_{j} a_{2 j}=$ $\sum_{j} a_{2 j+1}=1$. Using the project method (see [2, 5]) one can easily show the following.

Proposition 2.2. The support of any convergent subdivision scheme associated with a finite nonnegative mask $a=\left\{a_{\alpha}: \alpha \in \mathbb{Z}^{s}\right\}$ has property $P$.

Let $\mathcal{M}_{s}$ be the set of $s \times s$ unimodular matrices, namely,

$\mathcal{M}_{s}=\{M: M$ is an $s \times s$ matrix with integer entries and $|\operatorname{det} M|=1\}$.

Clearly, $\mathcal{M}_{s}$ is a group under the matrix production. In particular, $M \in \mathcal{M}_{s}$ implies $M^{-1} \in \mathcal{M}_{s}$. Clearly, there holds

Proposition 2.3. Let $\left\{a_{\alpha}: \alpha \in \mathbb{Z}^{s}\right\}$ be a finite mask in $\mathbb{R}^{s}$ and satisfy (1.3). Further, let $b_{\alpha}=a_{M \alpha}$ for any given $M \in \mathcal{M}_{s}$. Then, $\left\{b_{\alpha}\right\}$ satisfies the sum rule. Moreover, the convergence behavior of the subdivision schemes associated with $\left\{a_{\alpha}\right\}$ and $\left\{b_{\alpha}\right\}$ respectively are the same. 
Now let $X=\left\{x_{1}, \ldots, x_{\sigma}\right\} \subset \mathbb{Z}^{s} \backslash\{0\}$ be given and $\sigma \geq s$. We may regard $X$ as an $s \times \sigma$ matrix. The centered zonotope generated by the vectors in $X$ is defined by

$$
Z(X)=\left\{X u: u \in \mathbb{R}^{\sigma},-1 \leq(u)_{j} \leq 1, j=1, \ldots, \sigma\right\} .
$$

We say $X$ is unimodular if the determinant of each $s \times s$ submatrix of $X$ is $-1,0$ or 1. The following is proven by Cavaretta, Dahmen and Micchelli in 1991 (see [2]).

Theorem 2.4. Let $X=\left\{x_{1}, \ldots, x_{\sigma}\right\} \subset \mathbb{Z}^{s} \backslash\{0\}$ with $\sigma>s$. If the support of a nonnegative mask $\left\{a_{\alpha}\right\}$ is $Z(X) \cap \mathbb{Z}^{s}$ with unimodular $X$, then the corresponding subdivision scheme is convergent, provided the sum rule (1.3) for $\left\{a_{\alpha}\right\}$ is fulfilled.

Consequently, the question was raised in 2] (see p. 55 of [2]) as to what are the necessary and sufficient conditions on zonotopes such that the subdivision scheme with a nonnegative mask, whose support is a centered zonotope, converges. In [7] it is proven that, if the matrix $X$ is unimodular and $X \backslash\left\{x_{i}\right\}$ spans $\mathbb{R}^{s}$ for every $i=1, \ldots, \sigma$, then the convergence follows, provided the support of the nonnegative mask is $Z^{\prime}(X) \cap \mathbb{Z}^{s}$ and the sum rule is fulfilled, where

$$
Z^{\prime}(X)=\left\{X u: u \in \mathbb{R}^{\sigma}, 0 \leq(u)_{j} \leq 1, j=1, \ldots, \sigma\right\} .
$$

For centered zonotopes this problem is now solved. We have

Theorem 2.5. Let $X=\left\{x_{1}, \ldots, x_{\sigma}\right\} \subset \mathbb{Z}^{s} \backslash\{0\}$ with $\sigma \geq s$. Let the support $\Omega$ of a nonnegative mask $\left\{a_{\alpha}\right\}$ be $Z(X) \cap \mathbb{Z}^{s}$. Then the corresponding scheme is convergent if the sum rule (1.3) for $\left\{a_{\alpha}\right\}$ is fulfilled. Conversely, if $X$ is of rank $s$ one can always find a nonnegative mask $\left\{a_{\alpha}\right\}$, whose support is $Z(X) \cap Z^{s}$, and the sum rule for $\left\{a_{\alpha}\right\}$ is fulfilled.

We know that the sum rule is satisfied for any convergent subdivision scheme. It is little surprising that for centered zonotops one needs no additional condition. For noncentered zonotopes we can prove

Theorem 2.6. Let the support $\Omega$ of a nonnegative mask $\left\{a_{\alpha}\right\}$ be $Z^{\prime}(X) \cap \mathbb{Z}^{s}$. Assume for some $s \times s$ submatrix $Y$ of $X$ there holds $|\operatorname{det} Y|=1$. Then the corresponding scheme is convergent if and only if the sum rule for $\left\{a_{\alpha}\right\}$ is fulfilled and $Y^{-1} \Omega$ has property $P$.

In the above theorem the $s \times s$ submatrix $Y$ is clearly unimodular and belongs to $\mathcal{M}_{s}$. From Theorem 1.1 and Proposition 2.2 we know that for convergent subdivision schemes with nonnegative finite masks the two conditions are clearly fulfilled. It is unclear if the additional condition $|\operatorname{det} Y|=1$ on $Z^{\prime}(X)$ is redundant. Theorems 2.5 and 2.6 will be proven in Section 4 .

\section{Characterization of CONVERGEnt SUBDivision SCHEMES VIA MAPS GENERATED BY MASKS}

In this section we should establish a connection between convergent subdivision schemes and maps deduced by masks as in Wang [11. To this end, for any $m=$ $\left(m_{1}, \ldots, m_{s}\right)^{T}, n=\left(n_{1}, \ldots, n_{s}\right)^{T} \in \mathbb{Z}^{s}$ such that $m_{i}<n_{i}$, let

$$
\Gamma_{m, n}=\left\{\alpha \in \mathbb{Z}^{s}: \alpha=\left(\alpha_{1}, \ldots, \alpha_{s}\right)^{T}, m_{i} \leq \alpha_{i} \leq n_{i}, i=1, \ldots, s\right\} .
$$

Setting $n+1=\left(n_{1}+1, \ldots, n_{s}+1\right)$ we may assume that the support of the mask $a=\left\{a_{\alpha}: \alpha \in \mathbb{Z}^{s}\right\}$ is contained in $\Gamma_{m, n+1}$. Let $N=\prod_{i=1}^{s}\left(n_{i}-m_{i}+1\right)$ and define 
for every $e \in E_{s}$ the $N \times N$ matrix $A_{e}$ whose entries are

$$
\left(A_{e}\right)_{\alpha, \beta}=a_{e+2 \beta-\alpha}, \quad \alpha, \beta \in \Gamma_{m, n} .
$$

For any $N \times N$ matrix we should index the rows and columns by $\alpha, \beta \in \Gamma_{m, n}$. We note also that if the mask $a$ is nonnegative and satisfies the sum rule

$$
\sum_{\beta} a_{\alpha+2 \beta}=1, \quad \forall \alpha \in \mathbb{Z}^{s},
$$

then $A_{e}$ is a row stochastic matrix for all $e \in E_{s}$. We now have another characterization of the convergence:

Theorem 3.1. The subdivision scheme associated with a mask $a=\left\{a_{\alpha}: \alpha \in \mathbb{Z}^{s}\right\}$, which satisfies (3.2), is convergent if and only if for all $x=\left\{x_{\alpha}: \alpha \in \Gamma_{m, n}\right\}$ and all sequences $\left\{e_{j}\right\}, e_{j} \in E_{s}$,

$$
\lim _{k \rightarrow \infty} \Delta A_{e_{k}} \cdots A_{e_{1}} x=0,
$$

where $\Delta x=\max _{\mu, \nu}\left|x_{\mu}-x_{\nu}\right|$.

Proof. For $s=1$ this assertion is established in [1]. To show the assertion for $s \geq 2$ we apply the approach introduced in [4 (see also [6]). Let $S$ be the subdivision operator generated by $(S v)_{\alpha}=\sum_{\beta} v_{\beta} a_{\alpha-2 \beta}$, where $v=\left\{v_{\alpha}: \alpha \in \mathbb{Z}^{s}\right\}$ is any finite sequence. Further, let $J_{\alpha}$ be the shift map given by

$$
\left(J_{\alpha} v\right)_{\gamma}=v_{\alpha-\gamma}, \quad \gamma \in \Gamma_{m, n} .
$$

Thus, we have (see [6])

$$
J_{\alpha} S^{k}=A_{e_{k}} \cdots A_{e_{1}} J_{\alpha_{k}},
$$

where $\alpha=2^{k} \alpha_{k}+2^{k-1} e_{1}+\ldots+e_{k}$. We are now ready to prove the asserted equivalence. Assume first that the subdivision scheme associated with $\left\{a_{\alpha}\right\}$ is convergent. For any data $x=\left\{x_{\mu}: \mu \in \Gamma_{m, n}\right\}$ we write $x=J_{0} v$. Hence, with $\alpha=2^{k-1} e_{1}+\cdots+e_{k}$ we obtain for any $\beta \in \Gamma_{m, n}$

$$
\begin{aligned}
\left(A_{e_{k}} \cdots A_{e_{1}} x\right)_{\beta} & =\left(A_{e_{k}} \cdots A_{e_{1}} J_{0} v\right)_{\beta} \\
& =\left(J_{\alpha} S^{k} v\right)_{\beta}=v_{\alpha-\beta}^{k} .
\end{aligned}
$$

Noting that $\lim _{k \longrightarrow \infty}\left|\left(\alpha-\beta_{1}\right) / 2^{k}-\left(\alpha-\beta_{2}\right) / 2^{k}\right|=0$ for all $\beta_{1}, \beta_{2} \in \Gamma_{m, n}$ and $\alpha \in \mathbb{Z}^{s}$, we conclude

$$
\lim _{k \longrightarrow \infty} \Delta\left(A_{e_{k}} \cdots A_{e_{1}} x\right)=0 .
$$

For the other direction, we prove (1.4) according to Theorem 1.1. Assume for some $\beta_{1}, \beta_{2} \in \Gamma_{m, n}$ and $\alpha_{1} \in \mathbb{Z}^{s}$ that

$$
\sup _{\alpha \in \mathbb{Z}^{s}, e \in E_{s}}\left|a_{\alpha}^{k}-a_{\alpha-e}^{k}\right|=\left|a_{\alpha_{1}-\beta_{1}}^{k}-a_{\alpha_{1}-\beta_{2}}^{k}\right| .
$$

We choose $v=\delta, \delta=\left\{\delta_{0, \alpha}\right\}$. In this way we obtain for $\alpha_{1}=2^{k} \alpha_{k}+2^{k-1} e_{1}+\cdots+e_{k}$ and $j=1,2$

Thus,

$$
a_{\alpha_{1}-\beta_{j}}^{k}=\left(A_{e_{k}} \cdots A_{e_{1}} J_{\alpha_{k}} \delta\right)_{\beta_{j}} .
$$

$$
\sup _{\alpha \in \mathbb{Z}^{s}, e \in E_{s}}\left|a_{\alpha}^{k}-a_{\alpha-e}^{k}\right| \leq \Delta A_{e_{k}} \cdots A_{e_{1}} J_{\alpha_{k}} \delta .
$$

The desired assertion follows from the last inequality. 
Theorem 3.1 allows us to reduce the convergence problem to a fixed point problem of some maps. For any $T \subset \Gamma_{m, n}$ let $\chi_{T}$ be the vector $x=\left(x_{\alpha}\right)$ such that $x_{\alpha}=1$ if $\alpha \in T$ and zero otherwise. Next we define for any $N \times N$ matrix $B$

$$
F_{B}(T)=\left\{\alpha:\left(B \chi_{T}\right)_{\alpha}=1\right\}, \quad T \subseteq \Gamma_{m, n},
$$

and with the convenience $F_{e}=F_{A_{e}}$, where $A_{e}$ is given by (3.1). The following lemma for $s=1$ is proven in [11]. The proof for $s \geq 2$ is the same.

Lemma 3.2. Let $B$ be a nonnegative row stochastic matrix. Then

1) $\Delta B x \leq \Delta x$ and $F_{B}\left(T_{1}\right) \cap F_{B}\left(T_{2}\right)=\emptyset$ if $T_{1} \cap T_{2}=\emptyset$.

2) Let $C$ be another nonnegative row stochastic matrix. Then $F_{B C}=F_{B} \circ F_{C}$.

3) The subdivision scheme with nonnegative mask, which satisfies (3.2), diverges if and only if there exist disjoint proper subsets $T$ and $T^{\prime}$ of $\Gamma_{m, n}$ and a sequence $\left(e_{1}, \ldots, e_{k}\right), e_{l} \in E_{s}$ for some $k \geq 1$ such that

$$
T=F_{e_{k}} \circ \cdots \circ F_{e_{1}}(T) \text { and } T^{\prime}=F_{e_{k}} \circ \cdots \circ F_{e_{1}}\left(T^{\prime}\right) .
$$

Next let us define a map $\Psi$, that allows us to compute $F_{e}$ explicitly. To this end denote $\Omega(e)=\Omega \cap\left\{2 \mathbb{Z}^{s}+e\right\}$ for $e \in E_{s}$. This map is given as follows: for any $T \subset \mathbb{Z}^{s}$ let

$$
\Psi(T)=\bigcup_{e \in E_{s}}\left\{\bigcap_{\beta \in \Omega(e)}(2 T-\beta)\right\} .
$$

The connection between $\Psi$ and $F_{e}$ is established in the following lemma. We note that $s=1$ was proven in [1], where the support of the mask is $\{0, \ldots, N\}$ and $\Gamma_{m, n}=\{0, \ldots, N-1\}$. Although for $s \geq 2$ the approach is similar, we should give the proof for convenience.

Lemma 3.3. For any $T \subset \Gamma_{m, n}$ we have

$$
F_{e}(T)=(\Psi(T)+e) \cap \Gamma_{m, n}, \quad \forall e \in E_{s} .
$$

Furthermore, for any $e_{l} \in E_{s}, l=1, \ldots, k$, there holds

$$
F_{e_{1}} \circ \cdots \circ F_{e_{k}}(T)=\left(\Psi^{k}(T)+\lambda\right) \cap \Gamma_{m, n},
$$

where $\lambda=\sum_{l=1}^{k} e_{l} 2^{l-1}$.

Proof. For given $e \in E_{s}$ and $\alpha \in \Gamma_{m, n}$ let $I_{\alpha}=\left\{\beta:\left(A_{e}\right)_{\alpha, \beta} \neq 0\right\}$. We claim that for some $e^{\prime} \in E_{s}$ such that $\alpha \equiv e^{\prime}-e(\bmod 2)$, there holds

$$
I_{\alpha}=\frac{\Omega\left(e^{\prime}\right)-e+\alpha}{2} \text {. }
$$

Indeed, by the definition of $A_{e}$ we conclude that $\beta \in I_{\alpha}$ if and only if $a_{e+2 \beta-\alpha} \neq 0$. But, $e+2 \beta-\alpha \equiv e^{\prime}(\bmod 2)$. Thus, $\beta \in I_{\alpha}$ if and only if $e+2 \beta-\alpha \in \Omega\left(e^{\prime}\right)$ or in other words $\beta \in\left(\Omega\left(e^{\prime}\right)-e+\alpha\right) / 2$. To show the first assertion we note that $\alpha \in F_{e}(T)$ if and only if $\sum_{\beta \in I_{\alpha}} x_{\beta} a_{e+2 \beta-\alpha}=1$, where $\left\{x_{\beta}\right\}=\chi_{T}$. Hence, $I_{\alpha} \subset T$. We obtain

$$
\alpha \in \bigcap_{\beta \in \Omega\left(e^{\prime}\right)}(2 T-\beta+e) .
$$

Consequently,

$$
F_{e}(T) \subseteq \bigcup_{e^{\prime} \in E_{s}} \bigcap_{\beta \in \Omega\left(e^{\prime}\right)}(2 T-\beta+e)=(\Psi(T)+e) .
$$


Now let $\alpha \in(\Psi(T)+e) \cap \Gamma_{m, n}$. Then for some $e^{\prime} \in E_{s}$ one has $\alpha \in \Gamma_{m, n}$ and $\alpha \in \bigcap_{\beta \in \Omega\left(e^{\prime}\right)}(2 T-\beta+e)$, which in turn implies $\alpha \in 2 T-\beta+e$ for all $\beta \in \Omega\left(e^{\prime}\right)$ or $\alpha+\Omega\left(e^{\prime}\right)-e \subset 2 T$. Thus $I_{\alpha} \subset T$. We obtain $\sum_{\beta \in I_{\alpha}} x_{\beta} a_{e+2 \beta-\alpha}=1$ or $\alpha \in F_{e}(T)$. Thus,

To show the second assertion we use induction on the numbers of compositions.

$$
\begin{aligned}
F_{e_{1}} \circ \cdots \circ F_{e_{k}}(T) & =\left(\Psi\left(F_{e_{2}} \circ \cdots \circ F_{e_{k}}(T)\right)+e_{1}\right) \cap \Gamma_{m, n} \\
& =\left(\Psi\left\{\left(\Psi^{k-1}(T)+\lambda^{\prime}\right) \cap \Gamma_{m, n}\right\}+e_{1}\right) \cap \Gamma_{m, n} \\
& =\left(\Psi^{k}(T)+2 \lambda^{\prime}+e_{1}\right) \cap \Gamma_{m, n} \\
& =\left(\Psi^{k}(T)+\lambda\right) \cap \Gamma_{m, n} .
\end{aligned}
$$

The following lemma allows us to choose $k$ and $\lambda$ explicitly, which leads to the computation of $T$ for some $\Omega$.

Lemma 3.4. Let $\left\{a_{\alpha}\right\}$ be a nonnegative mask and let its support be $\Omega$. If the corresponding subdivision scheme with nonnegative mask, which satisfies (3.2), is divergent, then there exist disjoint proper subsets $T$ and $T^{\prime}$ of $\Gamma_{m, n}$ such that

$$
T=\left(\Psi^{k}(T)+\lambda\right) \cap \Gamma_{m, n} \quad \text { and } \quad T^{\prime}=\left(\Psi^{k}\left(T^{\prime}\right)+\lambda\right) \cap \Gamma_{m, n} .
$$

In particular, we can choose $k=k^{\prime} l$ for some $k^{\prime} \geq 1$ and any fixed $l \geq l_{0} \geq 1$ such that $0 \leq(\lambda)_{j} \leq\left(2^{k}-1\right), j=1, \ldots, s$. Moreover, if $(\lambda)_{j} \neq 0,2^{k}-1$, then for some $0<\delta_{1}<\delta_{2}<1$ there holds

$$
\delta_{1} 2^{k} \leq(\lambda)_{j} \leq \delta_{2} 2^{k} .
$$

Proof. By Lemma 3.2 the divergence implies

$$
T=F_{e_{k^{\prime}}} \circ \cdots \circ F_{e_{1}}(T) \text { and } T^{\prime}=F_{e_{k^{\prime}}} \circ \cdots \circ F_{e_{1}}\left(T^{\prime}\right)
$$

for some $k^{\prime} \geq 1$ and $e_{j} \in E_{s}, j=1, \ldots, k^{\prime}$. Write $F=F_{e_{k^{\prime}}} \circ \cdots \circ F_{e_{1}}$; we conclude that for all $l \geq 1$,

$$
T=F^{l}(T) \quad \text { and } \quad T^{\prime}=F^{l}\left(T^{\prime}\right) .
$$

It follows from Lemma 3.3 that

$$
T=\left(\Psi^{k^{\prime} l}(T)+\lambda^{\prime}\right) \cap \Gamma_{m, n} \quad \text { and } \quad T^{\prime}=\left(\Psi^{k^{\prime} l}\left(T^{\prime}\right)+\lambda^{\prime}\right) \cap \Gamma_{m, n},
$$

where

$$
\begin{aligned}
\lambda^{\prime} & =\lambda+\lambda 2^{k^{\prime}}+\ldots+\lambda 2^{(l-1) k^{\prime}} \\
& =\lambda \sum_{i=0}^{l-1} 2^{i k^{\prime}}=\frac{\lambda}{2^{k^{\prime}}-1}\left(2^{k^{\prime} l}-1\right) .
\end{aligned}
$$

Thus, we can choose $l$ large enough to meet the restrictions. Finally, setting $k=k^{\prime} l$ and defining $\lambda$ to be $\lambda^{\prime}$, we obtain (3.4) from (3.5).

\section{Proof of Theorems 2.5 And 2.6}

The notations such as $\Omega, \Psi$ and $F_{e}$ have the same meaning as in Sections 1-3. The following result for $s=1$ was proven in [13]. 
Lemma 4.1. Let $T$ be a subset of $\mathbb{Z}^{s}$ and $\alpha \notin T$ and let $\epsilon_{i, \gamma} \in\{0,1\}$ such that

$$
\sum_{\gamma \in \Omega} \epsilon_{i, \gamma}=1, \forall i=1,2, \ldots, l \text {. }
$$

Then there holds

$$
2^{l} \alpha-\sum_{i=1}^{l} \sum_{\gamma \in \Omega} 2^{l-i} \epsilon_{i, \gamma} \gamma \notin \Psi^{l}(T) .
$$

Proof. For $l=1$ the assertion is clear since by the definition of $\Psi$ one always has $2 \alpha-\gamma \notin \Psi(T)$ for all $\gamma \in \Omega$. On the other hand, $\sum_{\gamma \in \Omega} \epsilon_{1, \gamma}=1$ if and only if $\epsilon_{1, \gamma^{\prime}}=1$ for some $\gamma^{\prime} \in \Omega$ and $\epsilon_{1, \gamma}=0$ for all $\gamma \in \Omega \backslash\left\{\gamma^{\prime}\right\}$. Thus

$$
2 \alpha-\sum_{\gamma \in \Omega} \epsilon_{1, \gamma} \gamma \notin \Psi(T) .
$$

The general case follows from induction on $l$.

For $k$ and $\lambda$ from Lemma 3.4 and $\epsilon_{i, \gamma} \in\{0,1\}$ from Lemma 4.1 let us define $B_{0}=\{\alpha\}$, where $\alpha \in \Gamma_{m, n}$, and for $l=0,1, \ldots$,

$$
B_{l+1}=\left\{2^{k} y+\lambda-\sum_{i=0}^{k-1} \sum_{\gamma \in \Omega} 2^{i} \epsilon_{i, \gamma} \gamma \in \Gamma_{m, n}: y \in B_{l}\right\} .
$$

Finally, denote

$$
\mathcal{B}(\alpha)=\bigcup_{l \geq 0} B_{l}
$$

We have

Lemma 4.2. Let $\left\{a_{\alpha}\right\}$ be a nonnegative mask and let its support be $\Omega$. If $\left\{a_{\alpha}\right\}$ satisfies (3.2), then $\mathcal{B}(\alpha)=\Gamma_{m, n}$, for some $\alpha \in \Gamma_{m, n}$, implies the convergence of the corresponding subdivision scheme.

Proof. Assume the subdivision scheme is divergent. Thus, according to Lemma 3.4 there exist disjoint proper subsets $T$ and $T^{\prime}$ of $\Gamma_{m, n}$ satisfying (3.4). From Lemma 4.1 the sets $T$ or $T^{\prime}$ are not contained in $\mathcal{B}(\alpha)$ whenever $\alpha$ does not belong to $T$ or $T^{\prime}$. Thus, $T$ or $T^{\prime}$ must be empty. Consequently, the subdivision scheme must be convergent.

We are now in the position to verify Theorems 2.5 and 2.6.

Proof of Theorem 2.5. The sum rule implies $e_{\tau}+2 \gamma_{\tau} \in \Omega$ for some $\gamma_{\tau} \in \mathbb{Z}^{s}$ and $\tau=1, \ldots, s$, where as usual $e_{\tau} \in E_{s}, \tau=1, \ldots, s$, is the coordinate vector. But $\operatorname{det}\left(e_{1}+2 \gamma_{1}, \ldots, e_{s}+2 \gamma_{s}\right) \equiv 1(\bmod 2)$, so the rank of $M X$ for any $M \in \mathcal{M}_{s}$ is $s$. We may re-arrange $X$ such that $\left(x_{1}, \ldots, x_{s}\right)=X^{\prime}$ is a regular square matrix. On the other hand, it is well-known that there exists some $M \in \mathcal{M}_{s}$ such that $M X^{\prime}$ is a lower triangular matrix. Moreover, $M X^{\prime}=\left(c_{i, j}\right)_{1 \leq i, j \leq s}$ satisfies $0 \leq c_{l, j}<c_{j, j}$ if $l>j$. Thus, Proposition 2.3 allows us to suppose that $X^{\prime}$ is already such a matrix.

Next let us verify $\mathcal{B}(\alpha)=\Gamma_{m, n}$ for some $\alpha \in \Gamma_{m, n}$. Thus, Lemma 4.2 ensures the convergence of this subdivision scheme. To this end, we first note that $X^{\prime}$ is regular, so there are $u_{i} \in \mathbb{R}$ satisfying

$$
\lambda=\sum_{i=1}^{s} u_{i} x_{i}
$$


It is evident that each $u_{i}$ can be expressed as $u_{i}=v_{i}+2^{k} \eta_{i}$, where $\left|v_{i}\right| \leq 2^{k-1}+1$ and $\eta_{i} \in \mathbb{Z}$. We conclude for $\alpha=-\sum_{i=1}^{s} \eta_{i} x_{i} \in \mathbb{Z}^{s}$ that

$$
\lambda=\sum_{i=1}^{s} v_{i} x_{i}-2^{k} \alpha
$$

Now, for $l=1, \ldots, s$ we have

$$
\left|(\alpha)_{l}\right|=\frac{\left|(\lambda)_{l}-\sum_{i=1}^{s} v_{i}\left(x_{i}\right)_{l}\right|}{2^{k}} \leq 1-\frac{1}{2^{k}}+\left(\frac{1}{2}+\frac{1}{2^{k}}\right) \sum_{i=1}^{s}\left(x_{i}\right)_{l} .
$$

Because $0 \leq(\lambda)_{l} \leq 2^{k}-1$ and $\left|v_{i}\right| \leq 2^{k-1}+1$, one has

$$
-\sum_{i=1}^{s}\left(x_{i}\right)_{l} \leq(\alpha)_{l}<\sum_{i=1}^{s}\left(x_{i}\right)_{l} \text {. }
$$

Hence, $\alpha \in \Gamma_{m, n}$. Let $y \in \Gamma_{m, n}$, so there are $w_{i} \in \mathbb{R}$ such that

$$
y=\sum_{i=1}^{s} w_{i} x_{i}
$$

On the other hand, $k$ is large and independent of $\Gamma_{m, n}$ (see Lemma 3.4); we can therefore assume $\left|w_{i}\right| \leq 2^{k-2}-1$ for all $y \in \Gamma_{m, n}$. Clearly,

$$
2^{k} \alpha+\lambda-y=\sum_{i=1}^{s}\left(v_{i}-w_{i}\right) x_{i}
$$

and $\left|v_{i}-w_{i}\right| \leq 3 \times 2^{k-2}$. Write $v_{i}-w_{i}=2 l_{i}+\xi_{i}$ for some $l_{i} \in \mathbb{Z}$ and $-1 \leq \xi_{i} \leq 1$. We obtain by expressing $2 l_{i}$ in the binary form $2 l_{i}=\sum_{j=1}^{k-1} \delta_{i, j} 2^{j}$

$$
2^{k} \alpha+\lambda-y=\sum_{j=1}^{k-1} 2^{j}\left(\sum_{i=1}^{s} \delta_{i, j} x_{i}\right)+\sum_{i=1}^{s} \xi_{i} x_{i} .
$$

Clearly, $\gamma_{j}=\sum_{i=1}^{s} \delta_{i, j} x_{i} \in Z(X) \cap \mathbb{Z}^{s}$. Consequently, $\gamma_{0}=\sum_{i=1}^{s} \xi_{i} x_{i} \in Z(X) \cap \mathbb{Z}^{s}$. We obtain $2^{k} \alpha+\lambda-y=\sum_{j=0}^{k-1} 2^{j} \gamma_{j}$. This gives by the definition of $\mathcal{B}(\alpha)$ :

$$
y=2^{k} \alpha+\lambda-\sum_{j=0}^{k-1} 2^{j} \gamma_{j} \in \mathcal{B}(\alpha) .
$$

By Lemma 4.2 the scheme is convergent.

To show the second assertion, let $X=\left\{x_{1}, \ldots, x_{\sigma}\right\} \subset \mathbb{Z}^{s} \backslash\{0\}$ with $\sigma \geq s$ be of rank $s$. We may assume $\operatorname{det}\left(x_{1}, \ldots, x_{s}\right) \neq 0$. Thus, for each $e \in E_{s}$ there are $v_{i} \in \mathbb{R}$ such that

$$
e=\sum_{i=1}^{s} v_{i} x_{i}
$$

Write $v_{i}=2 l_{i}+\xi_{i}$ for some $l_{i} \in \mathbb{Z}$ and $-1 \leq \xi_{i} \leq 1$. We get $e=2 \sum_{i=1}^{s} l_{i} x_{i}+$ $\sum_{i=1}^{s} \xi_{i} x_{i}$, hence,

In other words,

$$
\sum_{i=1}^{s} \xi_{i} x_{i}=e-2 \sum_{i=1}^{s} l_{i} x_{i} \in Z(X) \cap \mathbb{Z}^{s} .
$$

$$
\left\{e+2 \beta: \beta \in \mathbb{Z}^{s}\right\} \cap Z(X) \neq \emptyset, \quad \forall e \in E_{s} .
$$


Having this relation we simply define

$$
a_{\alpha}= \begin{cases}\frac{1}{\left\{e+2 \beta: \beta \in \mathbb{Z}^{s}\right\} \cap Z(X) \mid}, & \text { if } \alpha=e+2 \gamma \text { and } \alpha \in Z(X) \cap \mathbb{Z}^{s} ; \\ 0, & \text { if } \alpha \notin Z(X) \cap \mathbb{Z}^{s} .\end{cases}
$$

So the sum rule (1.3) is fulfilled for the nonnegative mask $\left\{a_{\alpha}: \alpha \in \mathbb{Z}^{s}\right\}$ and $\left\{\alpha: a_{\alpha} \neq 0\right\}=Z(X) \cap \mathbb{Z}^{s}$.

Proof of Theorem 2.6. The necessity follows from Theorem 1.1 and Propositions 2.2 and 2.3. To show the sufficiency we remember $|\operatorname{det} Y|=1$. So $M=Y^{-1} \in \mathcal{M}_{s}$. By Proposition 2.3 we need only to show the convergence for the mask $b_{\alpha}=a_{M \alpha}$. We note that $M Z^{\prime}(X)=Z^{\prime}(M X)$ and the support of $\left\{b_{\alpha}\right\}$ is $M \Omega$. Moreover, $M Y=\left(e_{1}, \ldots, e_{s}\right)$. Thus, we can write $M X=\left(e_{1}, \ldots, e_{s}, x_{1}, \ldots, x_{\sigma-s}\right) . M \Omega$ has property P. Hence, we obtain in particular that $\sigma>s$ and $E_{s}+x_{l_{1}}+\cdots+x_{l_{\mu}} \subseteq M \Omega$ for any $1 \leq l_{1}<\cdots<l_{\mu} \leq \sigma-s$. Moreover, due to property $\mathrm{P}$ there is for each $1 \leq \tau \leq s$ some nonzero integer $p_{\tau}$ in $\left(Z^{\prime}\left(X_{1}\right) \cap \mathbb{Z}^{s}\right)_{\tau}$, where $X_{1}=\left(x_{1}, \ldots, x_{\sigma-s}\right)$. Consequently, for each $1 \leq \tau \leq s$ there is an $x_{i}$ with $1 \leq i \leq \sigma-s$ satisfying $\left(x_{i}\right)_{\tau} \neq 0$. Hence, we can find some nonnegative integers $j_{\nu}, \nu=1, \ldots, \sigma-s$, so that

$$
(x)_{\tau}=\left(\sum_{\nu=1}^{\sigma-s} j_{\nu} x_{\nu}\right)_{\tau} \neq 0, \quad \forall \tau=1,2, \ldots, s .
$$

In view of Lemma 4.2 , in order to have the desired assertion it is enough to verify that for $\Gamma_{m, n}$ defined by $M \Omega$ (see Section 3) and $B_{j}$ given by (4.1), there is an $\alpha \in$ $\Gamma_{m, n}$ such that $\mathcal{B}(\alpha)=\Gamma_{m, n}$. To this end, we remember that $\Gamma_{m, n}=I_{1} \times \cdots \times I_{s}$, where $I_{\tau}=\left[m_{\tau}, n_{\tau}\right], m_{\tau}=\min _{\gamma \in M \Omega}(\gamma)_{\tau}$ and $n_{\tau}=\max _{\gamma \in M \Omega}(\gamma)_{\tau}-1$. Clearly, $m_{\tau} \leq 0$ and $n_{\tau} \geq 0$. Moreover, if $(x)_{\tau}>0$, then $n_{\tau}>0$ and $m_{\tau}<0$ whenever $(x)_{\tau}<0$. Let $k$ and $\lambda$ be given by Lemma 3.4. We set $\alpha$ to be

$$
(\alpha)_{\tau}= \begin{cases}0, & (\lambda)_{\tau}=2^{k}-1 \text { and }(x)_{\tau}>0 ; \\ -1, & (\lambda)_{\tau}=2^{k}-1 \text { and }(x)_{\tau}<0 ; \\ 0, & (\lambda)_{\tau} \neq 0,2^{k}-1 ; \\ 1, & (\lambda)_{\tau}=0 \text { and }(x)_{\tau}>0 ; \\ 0, & (\lambda)_{\tau}=0 \text { and }(x)_{\tau}<0 .\end{cases}
$$

The above discussion implies $\alpha \in \Gamma_{m, n}$. Let $y \in \Gamma_{m, n}$. The two inequalities concerning $\lambda$ in Lemma 3.4 tell us that there are $\mu \in \mathbb{Z}^{s}$ and $l \in \mathbb{Z}$ satisfying $y=2^{k} \alpha+\lambda-\mu-l x$. Moreover, we can choose $l$ and $\mu$ such that $0 \leq l j_{\nu} \leq 2^{k}-1$ for $\nu=1, \ldots, \sigma-s$ and $0 \leq(\mu)_{\tau} \leq 2^{k}-1$ for $\tau=1, \ldots, s$. By expressing $\mu$ and $l j_{\nu}$ in the binary form we obtain for some $\epsilon_{i, \nu} \in\{0,1\}$ and $\delta_{i, \tau} \in\{0,1\}$

$$
l j_{\nu}=\sum_{i=0}^{k-1} \epsilon_{i, \nu} 2^{i} \text { and }(\mu)_{\tau}=\sum_{i=0}^{k-1} \delta_{i, \tau} 2^{i}
$$

Thereby,

$$
y=2^{k} \alpha+\lambda-\sum_{i=0}^{k-1} 2^{i}\left(\sum_{\tau=1}^{s} \delta_{i, \tau} e_{\tau}+\sum_{\nu=1}^{\sigma-s} \epsilon_{i, \nu} x_{\nu}\right)
$$


where $e_{\tau} \in E_{s}$ is the $\tau$-th coordinate vector. We deduce that for some $\gamma_{i} \in$ $\left(Z^{\prime}(M X) \cap \mathbb{Z}^{s}\right) \backslash\{0\}$ and $\epsilon_{i, \gamma_{i}}^{\prime} \in\{0,1\}$,

$$
\sum_{\tau=1}^{s} \delta_{i, \tau} e_{\tau}+\sum_{\nu=1}^{\sigma-s} \epsilon_{i, \nu} x_{\nu}=\epsilon_{i, \gamma_{i}}^{\prime} \gamma_{i}
$$

or

$$
y=2^{k} \alpha+\lambda-\sum_{i=0}^{k-1} 2^{i} \epsilon_{i, \gamma_{i}}^{\prime} \gamma_{i}
$$

Thereby, with $B_{0}=\{\alpha\}$ we obtain $\Gamma_{m, n} \subseteq B_{1}$, which in turn implies $\Gamma_{m, n}=\mathcal{B}(\alpha)$.

Consequently, the subdivision scheme with $\left\{b_{\alpha}\right\}$ is convergent.

\section{ACKNOWLEDGEMENT}

The author is indebted to the referee for various helpful comments on his paper.

\section{REFERENCES}

[1] M. Bröker and X. Zhou, Characterization of continuous, four-coefficient scaling functions via matrix spectral radius, SIAM J. Matrix Anal. Appl. 22 (2000), 242-257. MR1779727 (2001h:65169)

[2] A. S. Cavaretta, W. Dahmen, and C.A. Micchelli, Stationary Subdivision, Mem. Amer. Math. Soc. 453 (1991). MR.1079033(92h:65017)

[3] I. Daubechies and J. C. Lagarias, Two-scale difference equations I. Existence and global regularity of solutions, SIAM J. Math. Anal. 22 (1991), 1388-1410. MR1112515 (92d:39001)

[4] T. N. T. Goodman, C. M. Micchelli and J. Ward, Spectral radius formulas for subdivision operators, Recent advances in Wavelet Analysis, L.L. Schumaker and G. Webb (eds.), Academic Press, Inc. 1994, 335-360. MR:1244611 (94m:47076)

[5] B. Han, Projectable multivariate refinable functions and biorthogonal wavelets, Appl. Comput. Harmon. Anal. 13 (2002), 89-102. MR.1930178 (2003f:42054)

[6] B. Han and R.-Q. Jia, Multivariate refinement equations and convergence of subdivision schemes, SIAM J. Anal. 29 (1998), 1177-1199. MR1618691 (99f:41018)

[7] R.-Q. Jia and D.-X. Zhou, Convergence of subdivision schemes associated with nonnegative masks, SIAM J. Matrix Anal. Appl. 21 (1999), 418-430. MR1718338(2001a:42041)

[8] A. A. Melkman, Subdivision schemes with non-negative masks always converge - unless they obviously cannot? Ann. Numer. Math. 4 (1997), 451-460. MR1422696 (97i:41014)

[9] C. A. Micchelli and H. Prautzsch, Uniform refinement of curves, Linear Algebra Appl. 114/115 (1989), 841-870. MR0986909 (90k:65088)

[10] J. N. Tsitsiklis and V. D. Blondel, The Lyapunov exponent and joint spectral radius of pairs of matrices are hard, when not impossible, to compute and to approximate, Mathematics of Control, Signals and Systems 10 (1997), 31-41. MR1462278(99h:65238a)

[11] Y. Wang, Subdivision schemes and refinement equations with nonnegative masks, J. Approx. Th. 113 (2001), 207-220. MR1876323 (2002i:42054)

[12] X. Zhou, Characterization of convergent subdivision schemes, J. Approx. and its Appl. 14 (1998), 11-24. MR1668983 (99m:65041)

[13] X. Zhou, Subdivision schemes with nonnegative masks, Math. Comp. 74 (2005), 819-839. MR2114650

Department of Mathematics, China Jiliang University, 310018 Hangzhou, People's Republic of China - and - Department of Mathematics, University of Duisburg-Essen, D-47057 Duisburg, Germany

E-mail address: zhou@math.uni-duisburg.de 